\title{
On optimal classes of estimators in the presence of some non- sampling errors
}

\author{
M Javed", M Irfan and SH Bhatti \\ Department of Statistics, Government College University, Faisalabad, Pakistan.
}

\begin{abstract}
During a survey study, an investigator may be incapable of assembling the complete response (i.e., there is non-response) and/or the assembled response is not $100 \%$ true (i.e., measurement errors exist). In this situation, estimation of the population mean under stratified random sampling is not an easy task. Mostly these non-sampling errors, i.e. non-response and measurement error, significantly affect the estimators than sampling errors. To deal with this task, a progressive generalized estimator has been proposed, that can generate a number of estimators based on the availability of conventional and/or non-conventional auxiliary information. Ratio-type, ratio-type exponential, ratio-ratio-type exponential, ratio-product-type exponential, product-type, product-type exponential, product-product-type exponential and productratio-type exponential estimators are generated through the proposed generalized estimator. Mathematical properties such as bias, mean squared error and minimum mean squared error of the proposed estimator are derived up to first degree of approximation. The empirical performance of all the estimators in terms of percent relative efficiency is evaluated with the help of a simulation study. It turned out that the proposed estimators outperform when compared with Hansen and Hurwitz (1946) estimator and other competing estimators in this study i.e. Singh and Kumar's (2008), Kumar et al. (2015), Azeem and Hanif (2017) and Zahid and Shabbir (2018). It is suggested that the proposed estimators will be applied in case of non-response and measurement errors under stratified random sampling.
\end{abstract}

Keywords: Auxiliary information, non-sampling errors, percent relative efficiency, stratified random sampling.

\section{INTRODUCTION}

In a survey study, a researcher must be concerned about all sources of errors despite a vigilant survey is designed and conducted. These errors may have significant impact on the estimation of population parameters and can be broadly categorized as sampling and nonsampling errors. Sampling error (divergence among the estimate obtained from sample and the true value of the population parameter) has the privileged characteristic of being controllable by the design of sample and the size of sample. Non-sampling errors may be listed as: defective sampling procedures, ambiguity in definitions, faulty measurement techniques, mistakes in recording, incomplete coverage of sample units (non-response) and measurement errors etc. It is important to consider that with the increase of sample size; non-sampling errors increase.

This study emphasises on the estimation of population mean under stratified random sampling in the presence of two major non-sampling errors, i.e. non-response and measurement errors. Non-response refers to a situation when an investigator is incapable of gathering the pertinent information regarding some units of the population. The reasons behind this incapability may be, the respondents are not available at home, the

${ }^{*}$ Corresponding author (mariajaved@gcuf.edu.pk; (iD https://orcid.org/0000-0001-5468-8697) 
respondents are unapproachable, refusal of respondents, etc. Non-response is dominantly seen in surveys based on mails rather than in interviews conducted personally. Maximum information can be obtained by contacting the non-respondents to control this incomplete coverage. Hansen and Hurwitz (1946), Cochran (1977), Rao (1986), Singh and Kumar (2008), Singh et al. (2010), Kumar and Chatterjee (2014), Kumar (2015) and others carried out their efforts for the estimation of population parameters under simple random sampling in the presence of nonresponse.

The difference between recorded value and the true value of a variable is known as measurement error (ME). This concept may be clarified through an example. Let us assume a study associated with a person's age/weight. On investigation, it is possible that the person (respondent) does not remember his/her exact age/weight or may report a rounded figure or may tell a lie in place of his/her exact age/weight. Here the discrepancy among reported and exact age/weight is the measurement error. Cochran (1968; 1977), Shalabh (1997), Singh and Karpe (2009; 2010), Shukla et al. (2012) and Sharma and Singh (2013) contributed for the estimation of population mean under the influence of measurement errors.

Continuing the above efforts, Kumar et al. (2015), Kumar (2016), Azeem and Hanif (2017) and Irfan et al. (2018) suggested new estimators for population mean in simple random sampling. They considered the combined effect of non-response and measurement error. Zahid and Shabbir (2018) suggested a new estimator for the estimation of population mean under stratified random sampling. Their work is based on only conventional auxiliary information.

In context of above scenario, the present study proposed an optimal generalized estimator for the population mean under stratified random sampling without replacement by considering the joint influence of non-response and measurement error. The novelty behind this work is:

(i) Non-conventional auxiliary information as well as conventional auxiliary information is utilized for the first time for progressive estimation under stratified random sampling.

(ii) A variety of estimators can be generated through the proposed generalized estimator: ratio-type, ratiotype exponential, ratio-ratio-type exponential, ratioproduct-type exponential, product-type, product-type exponential, product-product-type exponential and product-ratio-type exponential.

\section{Some important assumptions and background}

(i) It is assumed that the measurement errors related to study variable and auxiliary variable are purely random;

(ii) Above said errors are uncorrelated with mean zero and variances $S_{U}^{2}$ and $S_{V}^{2}$;

(iii) As per Singh and Karpe (2009), no relationship exists among the true values of the variables and the measurement errors;

(iv) As per Hansen and Hurwitz (1946), the subsample of non-respondents responded when the investigator again contacted them through interview (in case of non-response);

(v) It is also supposed that there is an independence between measurement errors and/or non-responses for both variables under study.

Let us consider a finite population $U=\left\{U_{1}, U_{2}, U_{3}, \ldots, U_{N}\right\}$ of size $N$ and it can be stratified into $L$ homogenous strata with $h^{\text {th }}$ stratum containing $N_{h},(h=1,2, \ldots, L)$ units subject to the restriction that $\sum_{h=1}^{L} N_{h}=N$. Assume that each stratum consists of two mutually exclusive groups; $N_{h}=N_{1 h}+N_{2 h}$, where $N_{1 h}$ are the respondents and $N_{2 h}$ are the nonrespondents. A sample of size $n_{h}$ is drawn under simple random sampling without replacement (SRSWOR) from $h^{\text {th }}$ stratum such that $\sum_{h=1}^{L} n_{h}=n$.

Let an investigator email the questionnaires to all $n$ individuals. In $h^{\text {th }}$ stratum, he/she receives a response from $n_{1 h}$ individuals (i.e., respondents) and $n_{2 h}$ individuals does not respond (i.e., non-respondents). The investigator again contacts a subsample $k_{h}=\frac{n_{2 h}}{j_{h}} ; j_{h}>1$ of $n_{2 h}$ non-respondents. According to assumption (iv), this subsample completely respond when inquiry is done telephonically or through personal interview.

Consider $\left(x_{i h}, y_{i h}\right)$ be the pair of values for auxiliary variable $X$ and study variable $Y$ on the $i t h$ unit of $h^{\text {th }}$ stratum. Let the true values and observed values of the variables $(X, Y)$ represent by $\left(x_{i h}^{*}, y_{i h}^{*}\right)$ and $\left(X_{i h}^{*}, Y_{i h}^{*}\right)$ respectively for $i^{\text {th }}\left(i=1,2, \ldots, n_{h}\right)$ unit in the $h^{\text {th }}$ stratum. A complete description of important measures related to study variable and auxiliary variable are given in Table 1. 
Table 1: Important measures associated with study variable and auxiliary variable

\begin{tabular}{|c|c|c|c|}
\hline Measure & & Study variable $Y$ & Auxiliary variable $X$ \\
\hline Measurement error (ME) & & $U_{i h}^{*}=y_{i h}^{*}-Y_{i h}^{*}$ & $V_{i h}^{*}=x_{i h}^{*}-X_{i h}^{*}$ \\
\hline Sample mean & & $\bar{y}_{h}=n_{h}{ }^{-1} \sum_{i=1}^{n_{h}} y_{i h}$ & $\bar{x}_{h}=n_{h}{ }^{-1} \sum_{i=1}^{n_{h}} x_{i h}$ \\
\hline \multirow{3}{*}{ 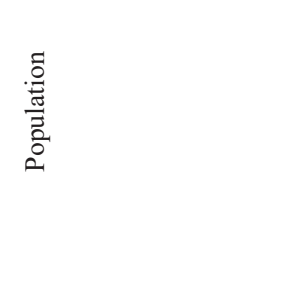 } & Mean & $\bar{Y}_{h}=N_{h}^{-1} \sum_{i=1}^{N_{h}} y_{i h}$ & $\bar{X}_{h}=N_{h}^{-1} \sum_{i=1}^{N_{h}} x_{i h}$ \\
\hline & Variance & $S_{Y h}^{2}=\frac{\sum_{i=1}^{N_{h}}\left(Y_{i h}-\bar{Y}_{h}\right)^{2}}{\left(N_{h}-1\right)}$ & $S_{X h}^{2}=\frac{\sum_{i=1}^{N_{h}}\left(X_{i h}-\bar{X}_{h}\right)^{2}}{\left(N_{h}-1\right)}$ \\
\hline & Variance associated with MEs & $S_{U h}^{2}=\frac{\sum_{i=1}^{N h}\left(U_{i h}-\bar{U}_{h}\right)^{2}}{\left(N_{h}-1\right)}$ & $S_{V h}^{2}=\frac{\sum_{i=1}^{N h}\left(V_{i h}-\bar{V}_{h}\right)^{2}}{\left(N_{h}-1\right)}$ \\
\hline \multirow{4}{*}{ 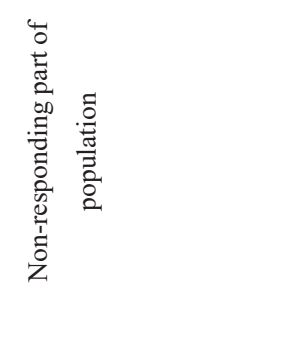 } & Coefficient of Variation & $C_{Y h}=\bar{Y}_{h}^{-1} S_{y h}$ & $C_{X h}=\bar{X}_{h}^{-1} S_{x h}$ \\
\hline & Variance & $S_{Y(2) h}^{2}=\frac{\sum_{i=1}^{N_{2 h}}\left(Y_{i h}-\bar{Y}_{h}\right)^{2}}{\left(N_{2 h}-1\right)}$ & $S_{X(2) h}^{2}=\frac{\sum_{i=1}^{N_{2 h}}\left(X_{i h}-\bar{X}_{h}\right)^{2}}{\left(N_{2 h}-1\right)}$ \\
\hline & Variance associated with MEs & $S_{U(2) h}^{2}=\frac{\sum_{i=1}^{N_{2 h}}\left(U_{i h}-\bar{U}_{h}\right)^{2}}{\left(N_{2 h}-1\right)}$ & $S_{V(2) h}^{2}=\frac{\sum_{i=1}^{N_{2 h}}\left(V_{i h}-\bar{V}_{h}\right)^{2}}{\left(N_{2 h}-1\right)}$ \\
\hline & Coefficient of Variation & $C_{Y(2) h}=\bar{Y}_{h}^{-1} S_{y(2) h}$ & $C_{X(2) h}=\bar{X}_{h}^{-1} S_{x(2) h}$ \\
\hline
\end{tabular}

\section{Transformation of existing estimators}

A review of literature reveals that the estimators for the estimation of population mean under joint effect of MEs and non-response are available only in simple random sampling. The current study has made transformation of all estimators under stratified random sampling.

The traditional Hansen and Hurwitz (1946) estimator along with its variance under stratified random sampling is given by

$$
\bar{y}_{s t(H H)}^{*}=\sum_{h=1}^{L} W_{h} \bar{y}_{h}^{*}
$$

where $\bar{y}_{h}^{*}=\left(\frac{n_{1 h}}{n_{h}}\right) \bar{y}_{n_{1 h}}+\left(\frac{n_{2 h}}{n_{h}}\right) \bar{y}_{k h}$ and $W_{h}=\frac{N_{h}}{N}$

$\operatorname{Var}\left(\bar{y}_{s t(H H)}^{*}\right)=\sum_{h=1}^{L} W_{h}^{2}\left[\lambda_{1 h}\left(S_{Y h}^{2}+S_{U h}^{2}\right)+\lambda_{2 h}\left(S_{Y(2) h}^{2}+S_{U(2) h}^{2}\right)\right]$

where $\lambda_{1 h}=\frac{\left(N_{h}-n_{h}\right)}{n_{h} N_{h}}$ and $\lambda_{2 h}=\frac{N_{2 h}\left(j_{h}-1\right)}{N_{h} n_{h}}$
Cochran's (1977) ratio-type estimator may be converted into stratified random sampling as below

$\bar{y}_{s t(C O)}=\sum_{h=1}^{L} W_{h} \frac{\bar{y}_{h}^{*}}{\bar{x}_{h}^{*}} \bar{X}_{h}$

Mean squared error (MSE) of $\bar{y}_{s t(C O)}$ is obtained as

$$
\begin{aligned}
M S E & \left(\bar{y}_{s t(C O)}\right) \cong \sum_{h=1}^{L} W_{h}^{2}\left[\lambda_{1 h} \bar{Y}_{h}^{2}\left(C_{Y h}^{2}+C_{X h}^{2}-2 \rho_{Y X h} C_{Y h} C_{X h}\right)\right. \\
& +\lambda_{2 h} \bar{Y}_{h}^{2}\left(C_{Y(2) h}^{2}+C_{X(2) h}^{2}-2 \rho_{Y X(2) h} C_{Y(2) h} C_{X(2) h}\right) \\
& \left.+\lambda_{1 h} \bar{Y}_{h}^{2}\left(\frac{S_{U h}^{2}}{\bar{Y}_{h}^{2}}+\frac{S_{V h}^{2}}{\bar{X}_{h}^{2}}\right)+\lambda_{2 h} \bar{Y}_{h}^{2}\left(\frac{S_{U(2) h}^{2}}{\bar{Y}^{2}}+\frac{S_{V(2) h}^{2}}{\bar{X}^{2}}\right)\right]
\end{aligned}
$$

Another ratio-type estimator was suggested by Rao (1986) in simple random sampling. Given below is the transformation of his estimator and its MSE in stratified random sampling 


$$
\begin{aligned}
& \bar{y}_{s t(R A)}=\sum_{h=1}^{L} W_{h} \frac{\bar{y}_{h}^{*}}{\bar{x}_{h}} \bar{X}_{h} \\
& \operatorname{MSE}\left(\bar{y}_{s t(R A)}\right) \cong \sum_{h=1}^{L} W_{h}^{2}\left[\lambda_{1 h} \bar{Y}_{h}^{2}\left(C_{Y h}^{2}+C_{X h}^{2}-2 \rho_{Y X h} C_{Y h} C_{X h}\right)\right. \\
& \left.+\lambda_{2 h} S_{Y(2) h}^{2}+\lambda_{1 h} \bar{Y}_{h}^{2}\left(\frac{S_{U h}^{2}}{\bar{Y}_{h}^{2}}+\frac{S_{V h}^{2}}{\bar{X}_{h}^{2}}\right)+\lambda_{2 h} S_{U(2) h}^{2}\right]
\end{aligned}
$$

Singh and Kumar's (2008) simple estimator is transformed into stratified random sampling in this way

$\bar{y}_{s t(S K)}=\sum_{h=1}^{L} W_{h} \bar{y}_{h}^{*}\left(\frac{\bar{X}_{h}}{\bar{x}_{h}^{*}}\right)\left(\frac{\bar{X}_{h}}{\bar{x}_{h}}\right)$

MSE of $\bar{y}_{s t(S K)}$ is given by

$$
\begin{aligned}
& M S E\left(\bar{y}_{S t(S K)}\right) \cong \sum_{h=1}^{L} W_{h}^{2}\left[\lambda_{1 h} \bar{Y}_{h}^{2}\left(C_{Y h}^{2}+4 C_{X h}^{2}-4 \rho_{Y X h} C_{Y h} C_{X h}\right)\right. \\
& +\lambda_{2 h} \bar{Y}_{h}^{2}\left(C_{Y(2) h}^{2}+C_{X(2) h}^{2}-2 \rho_{Y X(2) h} C_{Y(2) h} C_{X(2) h}\right) \\
& \left.+\lambda_{1 h} \bar{Y}_{h}^{2}\left(\frac{S_{U h}^{2}}{\bar{Y}_{h}^{2}}+4 \frac{S_{V h}^{2}}{\bar{X}_{h}^{2}}\right)+\lambda_{2 h} \bar{Y}_{h}^{2}\left(\frac{S_{U(2) h}^{2}}{\bar{Y}_{h}^{2}}+\frac{S_{V(2) h}^{2}}{\bar{X}_{h}^{2}}\right)\right]
\end{aligned}
$$

Kumar et al. (2015) suggested an exponential ratio type estimator in simple random sampling. Its conversion into stratified random sampling is given by

$$
\begin{aligned}
\bar{y}_{s t(K U)}= & \sum_{h=1}^{L} W_{h} \bar{y}_{h}^{*}\left(\frac{\bar{x}_{h}^{* *}}{\bar{X}_{h}}\right)\left[\alpha_{h} \exp \left(\frac{\bar{x}_{h}^{*}-\dot{\bar{x}}_{h}^{*}}{\bar{x}_{h}^{*}+\dot{\bar{x}}_{h}^{*}}\right)\right. \\
& \left.\left(1-\alpha_{h}\right) \exp \left(\frac{\dot{\bar{x}}_{h}^{*}-\bar{x}_{h}^{*}}{\overline{\bar{x}}_{h}^{*}+\bar{x}_{h}^{*}}\right)\right]
\end{aligned}
$$

where $\dot{\bar{x}}_{h}^{* *}=\frac{N_{h} \bar{X}_{h}-n_{h} \bar{x}_{h}}{N_{h}-n_{h}}, \dot{x}_{h}^{*}=\frac{N_{h} \bar{X}_{h}-n_{h} \bar{x}_{h}^{*}}{N_{h}-n_{h}}$

and $\alpha_{h}$ is the weight chosen in a way that minimizes the MSE. The weight of $\alpha_{h}$ and resulting MSE is determined as

$$
\begin{aligned}
& \alpha_{h}=\left(\frac{N_{h}+2 n_{h}}{2 N_{h}}\right)- \\
& \left(\frac{N_{h}-n_{h}}{N_{h}}\right)\left(\frac{\bar{X}_{h}}{\bar{Y}_{h}}\right)\left(\frac{\lambda_{1 h} \rho_{Y X h} S_{Y h} S_{X h}+\lambda_{2 h} \rho_{Y X(2) h} S_{Y(2) h} S_{X(2) h}}{\lambda_{1 h}\left(S_{X h}^{2}+S_{V h}^{2}\right)+\lambda_{2 h}\left(S_{X(2) h}^{2}+S_{V(2) h}^{2}\right)}\right)
\end{aligned}
$$

$$
\begin{aligned}
& M S E_{\min }\left(\bar{y}_{s t(K U)}\right) \\
& \cong \sum_{h=1}^{L} W_{h}^{2}\left[\lambda_{1 h}\left(S_{Y h}^{2}+S_{U h}^{2}\right)+\lambda_{2 h}\left(S_{Y(2) h}^{2}+S_{U(2) h}^{2}\right)\right. \\
& \left.\quad-\frac{\left(\lambda_{1 h} \rho_{Y X h} S_{Y h} S_{X h}+\lambda_{2 h} \rho_{Y X(2) h} S_{Y(2) h} S_{X(2) h}\right)^{2}}{\lambda_{1 h}\left(S_{X h}^{2}+S_{V h}^{2}\right)+\lambda_{2 h}\left(S_{X(2) h}^{2}+S_{V(2) h}^{2}\right)}\right]
\end{aligned}
$$

Azeem and Hanif (2017) proposed three estimators in simple random sampling. Given below are their modified versions in stratified random sampling

$$
\begin{aligned}
\bar{y}_{s t(A H 1)}= & \sum_{h=1}^{L} W_{h} \bar{y}_{h}^{*}\left(\frac{\dot{\bar{x}}_{h}^{*}}{\bar{X}_{h}}\right)\left(\frac{\dot{x}_{h}^{*}}{\bar{x}_{h}^{*}}\right) \\
\bar{y}_{s t(A H 2)=} & \sum_{h=1}^{L} W_{h} \bar{y}_{h}^{*}\left(\frac{\dot{x}_{h}^{*}}{\bar{X}_{h}}\right) \exp \left(\frac{\dot{\bar{x}}_{h}^{*}-\bar{x}_{h}^{*}}{\overline{\bar{x}}_{h}^{*}+\bar{x}_{h}^{*}}\right) \\
\bar{y}_{s t(A H 3)=} & \sum_{h=1}^{L} W_{h} \bar{y}_{h}^{*}\left[\alpha_{h} \exp \left(\frac{\bar{X}_{h}-\bar{x}_{h}^{*}}{\bar{X}_{h}+\bar{x}_{h}^{*}}\right)\right. \\
& \left.+\left(1-\alpha_{h}\right) \exp \left(\frac{\bar{x}_{h}^{*}-\bar{x}_{h}^{*}}{\overline{\bar{x}}_{h}^{*}+\bar{x}_{h}^{*}}\right)\right]
\end{aligned}
$$

where $\alpha_{h}$ is the same as stated above.

Following expression summarizes the MSEs of Azeem and Hanif's (2017) three estimators

$$
\begin{aligned}
& \operatorname{MSE}\left(\bar{y}_{s t(A H i)}\right) \cong \sum_{h=1}^{L} W_{h}^{2}\left[\lambda _ { 1 h } \overline { Y } _ { h } ^ { 2 } \left(C_{Y h}^{2}+\mu_{i h}^{2} C_{X h}^{2}\right.\right. \\
& \left.\quad-2 \mu_{i h} \rho_{Y X h} C_{Y h} C_{X h}\right)+\lambda_{2 h} \bar{Y}_{h}^{2}\left(C_{Y(2) h}^{2}+\mu_{i h}^{2} C_{X(2) h}^{2}\right. \\
& \left.\quad-2 \mu_{i h} \rho_{Y X(2) h} C_{Y(2) h} C_{X(2) h}\right)+\lambda_{1 h} \bar{Y}_{h}^{2}\left(\frac{S_{U h}^{2}}{\bar{Y}_{h}^{2}}+\mu_{i h}^{2} \frac{S_{V h}^{2}}{\bar{X}_{h}^{2}}\right) \\
& \left.+\lambda_{2 h} \bar{Y}_{h}^{2}\left(\frac{S_{U(2) h}^{2}}{\bar{Y}_{h}^{2}}+\mu_{i h}^{2} \frac{S_{V(2) h}^{2}}{\bar{X}_{h}^{2}}\right)\right] \text { for } i=1,2,3
\end{aligned}
$$

where $\mu_{1 h}=\frac{N_{h}+n_{h}}{N_{h}-n_{h}}, \mu_{2 h}=\frac{1}{2}\left(\frac{N_{h}+2 n_{h}}{N_{h}-n_{h}}\right)$ 
and $\mu_{3 h(o p t)}=\frac{\left[\lambda_{1 h} \rho_{Y X h} C_{Y h} C_{X h}+\lambda_{2 h} \rho_{Y X(2) h} C_{Y(2) h} C_{X(2) h}\right]}{\lambda_{1 h}\left(\frac{S_{X h}^{2}+S_{V h}^{2}}{\bar{X}_{h}^{2}}\right)+\lambda_{2 h}\left(\frac{S_{X(2) h}^{2}+S_{V(2) h}^{2}}{\bar{X}_{h}^{2}}\right)}$

Recently, Zahid and Shabbir (2018) proposed an estimator in stratified random sampling detailed below

$$
\begin{aligned}
& \bar{y}_{s t(Z S)}=\sum_{h=1}^{L} W_{h}\left[\left\{m_{1 h} \bar{y}_{h}^{*}+m_{2 h}\left(\bar{X}_{h}-\dot{x}_{h}^{*}\right)\right\}\left(\frac{\bar{X}_{h}}{\bar{x}_{h}^{*}}\right)^{\gamma_{h}}\right. \\
& \left.\exp \left(1-\gamma_{h}\right)\left(\frac{\bar{X}_{h}-\dot{x}_{h}^{*}}{\bar{X}_{h}+\dot{\bar{x}}_{h}^{*}}\right)\right]
\end{aligned}
$$

where $m_{1 h}$ and $m_{2 h}$ are the suitably chosen weights and $\gamma_{h}$ is the scalar, and takes the values $(-1,0,1)$.

Minimum MSE of $\bar{y}_{s t(Z S)}$ is given as

$$
\begin{aligned}
& \operatorname{MSE}_{\min }\left(\bar{y}_{s t(Z S)}\right) \cong \\
& \sum_{h=1}^{L} W_{h}^{2}\left[\bar{Y}_{h}^{2}-\frac{\left(M_{1 h} M_{5 h}^{2}+M_{2 h} M_{4 h}^{2}-2 M_{3 h} M_{4 h} M_{5 h}\right)}{\left(M_{1 h} M_{2 h}-M_{3 h}^{2}\right)}\right]
\end{aligned}
$$

where

$$
\begin{aligned}
& M_{1 h}=\bar{Y}_{h}^{2}+q_{2 h}+e_{h}^{2} t_{h}^{2} R_{h}^{2} q_{1 h}+4 e_{h} t_{h} R_{h} q_{3 h}+2 f_{h} t_{h}^{2} R_{h}^{2} q_{1 h}, \\
& M_{2 h}=t_{h}^{2} q_{1 h} \\
& M_{3 h}=t_{h} q_{3 h}+2 e_{h} t_{h}^{2} R_{h} q_{1 h}, \\
& M_{4 h}=\bar{Y}_{h}^{2}+e_{h} t_{h} R_{h} q_{3 h}+f_{h} t_{h}^{2} R_{h}^{2} q_{1 h} \text { and } \\
& M_{5 h}=e_{h} t_{h}^{2} R_{h} q_{1 h}
\end{aligned}
$$

here

$$
\begin{aligned}
& e_{h}=\frac{1+\gamma_{h}}{2}, \quad t_{h}=\frac{n_{h}}{N_{h}-n_{h}}, R_{h}=\frac{\bar{Y}_{h}}{\bar{X}_{h}} \quad \text { and } \\
& f_{h}=\frac{\gamma_{h}^{2}+4 \gamma_{h}+3}{8}
\end{aligned}
$$

\section{METHODOLOGY}

This section suggests a new generalized estimation for the population mean in stratified random sampling without replacement under the joint influence of measurement errors and non-response. The following generalized estimator is suggested.
$T_{s t}=\sum_{h=1}^{L} W_{h}\left[\omega_{1 h} \bar{y}_{h}^{*}\left(\frac{\bar{Z}_{h}}{\bar{z}_{h}}\right)^{g_{h}}+\omega_{2 h} \bar{y}_{h}^{*} \exp \left(\frac{\delta_{h}\left(\bar{Z}_{h}-\bar{z}_{h}\right)}{\bar{Z}_{h}+\bar{z}_{h}}\right)\right]$

where $\bar{Z}_{h}=a_{h} \bar{X}_{h}+b_{h}$ and $\bar{z}_{h}=a_{h} \dot{\bar{x}}_{h}^{*}+b_{h}$

and $a_{h}(\neq 0), b_{h}$ are either available conventional or nonconventional auxiliary information or any real number. $\left(\omega_{1 h}, \omega_{2 h}\right)$ are the suitably chosen weights that cause the MSE minimum. $\left(g_{h}, \delta_{h}\right)$ being constants assume values $(-1,0,1)$. These constants help us to generate a variety of estimators given in Table 2 (See Appendix).

Remark 1: Conventional parameters of the $h^{\text {th }}$ stratum may be listed as median $Q_{2 h}$, standard deviation $S_{X h}$, coefficient of variation $C_{X h}$, coefficient of skewness $\beta_{1 h}(X)$, coefficient of kurtosis $\beta_{2 h}(X)$ and coefficient of correlation $\rho_{Y X h}$ etc.

Remark 2: Non-conventional parameters of the $h^{\text {th }}$ stratum include quartile deviation $Q D_{h}$, mid-range $M R_{h}$, trimmed mean $T M_{h}$, Hodge-Lehmann estimator $H L_{h}$, etc. where

$$
\begin{aligned}
Q D_{h} & =\frac{Q_{3 h}-Q_{1 h}}{2}, \\
M R_{h} & =\frac{X_{(1) h}+X_{(N) h}}{2}, \\
T M_{h} & =\frac{Q_{1 h}+2 Q_{2 h}+Q_{3 h}}{4} \text { and } \\
H L_{h} & =\text { median }\left(\frac{X_{j h}+X_{k h}}{2}\right), 1 \leq j h \leq k h \leq N
\end{aligned}
$$

Remark 3: We can generate a number of estimators by substituting known parameters (given in remark 1 and remark 2) in $a_{h}$ and $b_{h}$ in $T_{s t i}^{(1)}$. Similar process can be adopted for $T_{s t i}^{(2)}, T_{s t i}^{(3)}, \ldots, T_{s t i}^{(8)}$ to gain many more estimators. Some of them $\left(T_{s t i}^{(1)}\right.$ and $\left.T_{s t i}^{(3)}\right)$ are presented in Table 3 (See Appendix).

It is necessary to describe some expressions to obtain the mathematical properties of the proposed estimator $T_{s t}$. These properties include bias, MSE and minimum MSE.

$$
\begin{aligned}
& \omega_{Y h}^{*}=\sum_{i=1}^{n_{h}}\left(Y_{i h}^{*}-\bar{Y}_{h}\right), \quad \omega_{X h}^{*}=\sum_{i=1}^{n_{h}}\left(X_{i h}^{*}-\bar{X}_{h}\right), \\
& \omega_{U h}^{*}=\sum_{i=1}^{n_{h}} U_{i h}^{*} \text { and } \omega_{V h}^{*}=\sum_{i=1}^{n_{h}} V_{i h}^{*}
\end{aligned}
$$

Adding $\omega_{Y h}^{*}$ and $\omega_{U h}^{*}$ dividing by $n_{h}$, we get 


$$
\begin{array}{cc}
\frac{1}{n_{h}}\left(\omega_{Y h}^{*}+\omega_{U h}^{*}\right)=\frac{1}{n_{h}} \sum_{i=1}^{n_{h}} y_{i h}^{*}-\bar{Y}_{h} & \text { In the same way, we have } \\
\bar{y}_{h}^{*}=\bar{Y}_{h}+\frac{1}{n_{h}}\left(\omega_{Y h}^{*}+\omega_{U h}^{*}\right) & \bar{x}_{h}^{*}=\bar{X}_{h}+\frac{1}{n_{h}}\left(\omega_{X h}^{*}+\omega_{V h}^{*}\right)
\end{array}
$$

After taking expectations, we have

The new generalized estimator given in equation (17) can be rewritten using equation (18) and equation (19) in the following way. Expression is taken up to first degree of approximation.

$$
\begin{aligned}
& T_{s t}-\bar{Y} \cong \sum_{h=1}^{L} W_{h}\left[\bar{Y}_{h}\left(\omega_{1 h}+\omega_{2 h}-1\right)+\left(\omega_{1 h}+\omega_{2 h}\right)\left(\frac{\omega_{Y h}^{*}+\omega_{U h}^{*}}{n_{h}}\right)+\bar{Y}_{h} \tau_{h}\left(\omega_{1 h} g_{h} n_{h}+\frac{1}{2} \omega_{2 h} \delta_{h}\right)\left(\frac{\omega_{X h}^{*}+\omega_{V h}^{*}}{n_{h}}\right)\right. \\
& \left.+\bar{Y}_{h} \tau_{h}^{2}\left(\frac{1}{4} \omega_{2 h} \delta_{h}\left(1+\frac{\delta_{h}}{2}\right)-\frac{1}{2} \omega_{1 h} n_{h}^{2} g_{h}\left(g_{h}+1\right)\right)\left(\frac{\omega_{X h}^{*}+\omega_{V h}^{*}}{n_{h}}\right)^{2}+\tau_{h}\left(\omega_{1 h} g_{h} n_{h}+\frac{1}{2} \omega_{2 h} \delta_{h}\right)\left(\frac{\omega_{Y h}^{*}+\omega_{U h}^{*}}{n_{h}}\right)\left(\frac{\omega_{X h}^{*}+\omega_{V h}^{*}}{n_{h}}\right)\right]
\end{aligned}
$$

where $\tau_{h}=\frac{a_{h}}{\left(N_{h}-n_{h}\right)\left(a_{h} \bar{X}_{h}+b_{h}\right)}$

The bias of $T_{s t}$ is obtained up to first degree of approximation in this way

$$
\begin{aligned}
& \operatorname{Bias}\left(T_{s t}\right)=E\left(T_{s t}-\bar{Y}\right) \\
& \operatorname{Bias}\left(T_{s t}\right) \cong \sum_{h=1}^{L} W_{h}\left[\bar{Y}_{h}\left(\omega_{1 h}+\omega_{2 h}-1\right)+\bar{Y}_{h} \tau_{h}^{2} q_{1 h}\left(\frac{1}{4} \omega_{2 h} \delta_{h}\left(1+\frac{\delta_{h}}{2}\right)-\frac{1}{2} \omega_{1 h} n_{h}^{2} g_{h}\left(g_{h}+1\right)\right)+\tau_{h} q_{3 h}\left(\omega_{1 h} g_{h} n_{h}+\frac{1}{2} \omega_{2 h} \delta_{h}\right)\right]
\end{aligned}
$$

The MSE of the new estimator $T_{s t}$ can be obtained by squaring both sides of equation (21) and taking the expectation $\operatorname{MSE}\left(T_{s t}\right) \cong \sum_{h=1}^{L} W_{h}^{2}\left[\bar{Y}_{h}+\omega_{1 h}^{2} A_{h}+\omega_{2 h}^{2} B_{h}-\omega_{1 h} C_{h}-\omega_{2 h} D_{h}+\omega_{1 h} \omega_{2 h} E_{h}\right]$

where

$$
\begin{aligned}
A_{h} & =\left[\bar{Y}_{h}-q_{1 h} \bar{Y}_{h}^{2} \tau_{h}^{2} n_{h}^{2} g_{h}+q_{2 h}+4 q_{3 h} \bar{Y}_{h} \tau_{h} n_{h} g_{h}\right] \\
B_{h} & =\left[\bar{Y}_{h}+\frac{1}{2} q_{1 h} \bar{Y}_{h}^{2} \tau_{h}^{2} \delta_{h}\left(1+\delta_{h}\right)+q_{2 h}+2 q_{3 h} \bar{Y}_{h} \tau_{h} \delta_{h}\right] \\
C_{h} & =\left[2 \bar{Y}_{h}-q_{1 h} \bar{Y}_{h}^{2} \tau_{h}^{2} n_{h}^{2} g_{h}\left(1+g_{h}\right)+2 q_{3 h} \bar{Y}_{h} \tau_{h} n_{h} g_{h}\right] \\
D_{h} & =\left[2 \bar{Y}_{h}+\frac{1}{2} q_{1 h} \bar{Y}_{h}^{2} \tau_{h}^{2} \delta_{h}\left(1+\frac{\delta_{h}}{2}\right)+q_{3 h} \bar{Y}_{h} \tau_{h} \delta_{h}\right] \\
E_{h} & =\left[2 \bar{Y}_{h}+q_{1 h} \bar{Y}_{h}^{2} \tau_{h}^{2}\left\{\frac{\delta_{h}}{2}\left(1+\frac{\delta_{h}}{2}\right)-n_{h}^{2} g_{h}\left(1+g_{h}\right)+\delta_{h} n_{h} g_{h}\right\}+2 q_{2 h}+4 q_{3 h} \bar{Y}_{h} \tau_{h}\left\{\frac{\delta_{h}}{2}+n_{h} g_{h}\right\}\right]
\end{aligned}
$$


The optimum weights of $\omega_{1 h}$ and $\omega_{2 h}$ are given as

$$
\begin{aligned}
\omega_{1 h(o p t)} & =\frac{2 B_{h} C_{h}-D_{h} E_{h}}{E_{h}^{2}-4 A_{h} B_{h}} \\
\omega_{2 h(o p t)} & =\frac{2 A_{h} D_{h}-C_{h} E_{h}}{E_{h}^{2}-4 A_{h} B_{h}}
\end{aligned}
$$

Minimum MSE is obtained by placing the optimum weights of $\omega_{1 h}$ and $\omega_{2 h}$ in equation (23)

$$
\begin{aligned}
M S E_{\min }\left(T_{s t}\right) \cong \\
\sum_{h=1}^{L} W_{h}^{2}\left[\bar{Y}_{h}-\frac{\left(C_{h} D_{h} E_{h}-B_{h} C_{h}^{2}-A_{h} D_{h}^{2}\right)}{\left(E_{h}^{2}-4 A_{h} B_{h}\right)}\right]
\end{aligned}
$$

\section{RESULTS AND DISCUSSION}

A simulation study is conducted in this section to assess the empirical performance of proposed estimators as compared to other estimators. Two populations are generated through multivariate normal distribution under different parameters with the help of $\mathrm{R}$ language programme. Population I is generated for equal strata and population II is generated for unequal strata. Data statistics of population I and population II are shown in Tables 4 and 5. Equation (25) presents an expression to compute the percent relative efficiencies (PREs) of all the estimators with respect to the Hansen and Hurwitz (1946).

$$
P R E=\frac{M S E\left(\bar{y}_{s t(H H)}^{*}\right)}{M S E(\odot)} \times 100
$$

where $\bigcirc=\bar{y}_{s t(H H)}^{*}, \bar{y}_{s t(K U)}, \bar{y}_{s t(A H i)}, \bar{y}_{s t(Z S)}, T_{s t i}^{(1)}$,

$T_{s t i}^{(2)}, T_{s t i}^{(3)}, T_{s t i}^{(4)}, T_{s t i}^{(5)}, T_{s t i}^{(6)}, T_{s t i}^{(7)}, T_{s t i}^{(8)}$

Ten percent $(10 \%)$ non-response is considered to calculate the PREs and are presented in Tables 6 and 7. Important findings from Tables 6 and 7 are summarized below.

- Kumar et al. (2015) and third estimator of Azeem and Hanif (2017) are equally efficient as in each case PRE of $\bar{y}_{s t(K U)}=$ PRE of $\bar{y}_{s t(A H 3)}$.
- $\bar{y}_{s t(Z S)}$ perform better than $\bar{y}_{s t(H H)}^{*}, \bar{y}_{s t(K U)}$ and $\bar{y}_{s t(A H i)}$. Moreover, $\bar{y}_{s t(Z S)}$ provides maximum PRE at $\gamma_{h}=0$ in each case.

- It is worth pointing out that all the proposed series of estimators $T_{s t i}^{(1)}, T_{s t i}^{(2)}, T_{s t i}^{(3)}, T_{s t i}^{(4)}, T_{s t i}^{(5)}, T_{s t i}^{(6)}, T_{s t i}^{(7)}, T_{\text {sti }}^{(8)}$ are more competent than $\bar{y}_{s t(Z S) \text {.. }}$

Therefore, the above findings confirmed that the proposed series of estimators out-perform than all competing estimators under study.

\section{CONCLUSION}

This paper emphasizes on the utilisation of nonconventional auxiliary information as well as conventional information for enhanced estimation of population mean in stratified random sampling under the combined effect of non-response and measurement error. Different series of ratio and product type estimators have been proposed in the present study. These series can generate a number of estimators by choosing different known auxiliary information (conventional and non-conventional). Expressions of bias and MSE of the proposed estimators are derived up to first degree of approximation. Using $\mathrm{R}$ language programme, a simulation study is conducted by generating two populations from multivariate normal distribution. First population is generated with equal strata and second population with unequal strata. Empirical performance of all the estimators under study is assessed in terms of PREs with respect to Hansen and Hurwitz (1946). Suggested estimators outperform than all other competing estimators. Therefore, in case of non-response and measurement errors under stratified random sampling it is suggested to apply the proposed estimators.

The possible extensions of this work are to estimate the: 1) finite population mean under other sampling designs like stratified double sampling, rank set sampling, etc. 2) other unknown finite population parameters including median, variance and proportions etc.

\section{REFERENCES}

Azeem M. \& Hanif M. (2017). Joint influence of measurement error and non-response on estimation of population mean. Communications in Statistics-Theory and Methods 46(4): 1679-1693.

DOI: https://doi.org/10.1080/03610926.2015.1026992

Cochran W. (1968). Errors of measurement in statistics. Technometrics 10(4): 637-666.

DOI: https://doi.org/10.2307/1267450

Cochran W. (1977). Sampling Techniques, $3^{\text {rd }}$ edition. John Wiley \& Sons, Inc., New York, USA. 
Hansen M.H. \& Hurwitz W.N. (1946). The problem of nonresponse in sample surveys. Journal of American Statistical Association 41: 517-529.

DOI: https://doi.org/10.1080/01621459.1946.10501894

Irfan M., Javed M. \& Lin Z. (2018). Optimized estimation for population mean using conventional and non-conventional measures under the joint influence of measurement error and non-response. Journal of Statistical Computation and Simulation 88(12): 2385-2403.

DOI: https://doi.org/10.1080/00949655.2018.1464571

Kumar S. (2015). Efficient use of auxiliary information in estimating the population ratio, product and mean in the presence of non-response. Journal of Advanced Computing 4(2): 59-67.

DOI: https://doi.org/10.7726/jac.2015.1005

Kumar S. (2016). Improved estimation of population mean in presence of nonresponse and measurement error. Journal of Statistical Theory and Practice 10(4): 707-720. DOI: https://doi.org/10.1080/15598608.2016.1216488

Kumar S., Bhougal S., Nataraja N. S. \& Viswanathaiah M. (2015). Estimation of population mean in the presence of non-response and measurement error. Revista Colombiana de Estadistica 38(1): 145-161. DOI: https://doi.org/10.15446/rce.v38n1.48807

Kumar S. \& Chatterjee K. (2014). A simulation study for unit non-response under double sampling. Journal of Statistics Applications and Probability Letters 2(1): 37-50.

Rao P. (1986). Ratio estimation with sub sampling the nonrespondents. Survey Methodology 12: 217-230.

Shalabh (1997). Ratio method of estimation in the presence of measurement errors. Journal of the Indian Society of Agricultural Statistics 50: 150-155.

Sharma P. \& Singh R. (2013). A generalized class of estimators for finite population variance in presence of measurement errors. Journal of Modern Applied Statistical Methods 12: 231-241.

DOI: https://doi.org/10.22237/jmasm/1383279120

Shukla D., Pathak S. \& Thakue N.S. (2012). Class(es) of factortype estimator(s) in presence of measurement error. Journal of Modern Applied Statistical Methods 11: 336-347. DOI: https://doi.org/10.22237/jmasm/1351742700

Singh H.P. \& Karpe N. (2009). On the estimation of ratio and product of two population means using supplementary information in presence of measurement errors. Statistica 69: $27-47$.

DOI: https://doi.org/10.6092/issn.1973-2201/3546

Singh H. P. \& Karpe N. (2010). Estimation of mean, ratio and product using auxiliary information in the presence of measurement errors in sample surveys. Journal of Statistical Theory and Practice 4: 111-136. DOI: https://doi.org/10.1080/15598608.2010.10411976

Singh H.P. \& Kumar S. (2008). A regression approach to the estimation of the finite population mean in the presence of non-response. Australian and New Zealand Journal of Statistics 50: 395-408.

DOI: https://doi.org/10.1111/j.1467-842X.2008.00525.x

Singh H.P., Kumar S. \& Kozak M. (2010). Improved estimation of finite population mean using sub-sampling to deal with the non-response in two-phase sampling scheme. Communications in Statistics-Theory and Methods 39(5): 791-802.

DOI: https://doi.org/10.1080/03610920902796056

Zahid E. \& Shabbir J. (2018). Estimation of population mean in the presence of measurement error and nonresponse under stratified random sampling. PLOS ONE 13(2): e0191572. DOI: https://doi.org/10.1371/journal.pone.0191572 


\section{Appendix}

Table 2: Generation of series with proposed estimator $T_{s t}$

\begin{tabular}{|c|c|c|c|c|c|}
\hline \multicolumn{4}{|c|}{ Values of $\left(\omega_{1 h}, \omega_{2 h}, g_{h}, \delta_{h}\right)$} & \multicolumn{2}{|l|}{ Series of estimators } \\
\hline$\omega_{1 h}$ & $\omega_{2 h}$ & $g_{h}$ & $\delta_{h}$ & Estimator & Name \\
\hline$\omega_{1 h}$ & $\omega_{2 h}$ & 1 & 0 & $T_{s t i}^{(1)}=\sum_{h=1}^{L} W_{h}\left[\omega_{1 h} \bar{y}_{h}^{*}\left(\frac{\bar{Z}_{h}}{\bar{Z}_{h}}\right)+\omega_{2 h} \bar{y}_{h}^{*}\right]$ & Ratio-type \\
\hline$\omega_{1 h}$ & $\omega_{2 h}$ & -1 & 0 & $T_{s t i}^{(2)}=\sum_{h=1}^{L} W_{h}\left[\omega_{1 h} \bar{y}_{h}^{*}\left(\frac{\bar{Z}_{h}}{\bar{Z}_{h}}\right)+\omega_{2 h} \bar{y}_{h}^{*}\right]$ & Product-type \\
\hline$\omega_{1 h}$ & $\omega_{2 h}$ & 0 & 1 & $T_{s t i}^{(3)}=\sum_{h=1}^{L} W_{h}\left[\omega_{1 h} \bar{y}_{h}^{*}+\omega_{2 h} \bar{y}_{h}^{*} \exp \left(\frac{\bar{Z}_{h}-\bar{z}_{h}}{\bar{Z}_{h}+\bar{z}_{h}}\right)\right]$ & Ratio-type exponential \\
\hline$\omega_{1 h}$ & $\omega_{2 h}$ & 0 & -1 & $T_{s t i}^{(4)}=\sum_{h=1}^{L} W_{h}\left[\omega_{1 h} \bar{y}_{h}^{*}+\omega_{2 h} \bar{y}_{h}^{*} \exp \left(\frac{\bar{z}_{h}-\bar{Z}_{h}}{\bar{Z}_{h}+\bar{z}_{h}}\right)\right]$ & Product-type exponential \\
\hline$\omega_{1 h}$ & $\omega_{2 h}$ & 1 & 1 & $T_{s t i}^{(5)}=\sum_{h=1}^{L} W_{h}\left[\omega_{1 h} \bar{y}_{h}^{*}\left(\frac{\bar{Z}_{h}}{\bar{z}_{h}}\right)+\omega_{2 h} \bar{y}_{h}^{*} \exp \left(\frac{\bar{Z}_{h}-\bar{z}_{h}}{\bar{Z}_{h}+\bar{z}_{h}}\right)\right.$ & Ratio-ratio-type exponential \\
\hline$\omega_{1 h}$ & $\omega_{2 h}$ & -1 & -1 & $T_{s t i}^{(6)}=\sum_{h=1}^{L} W_{h}\left[\omega_{1 h} \bar{y}_{h}^{*}\left(\frac{\bar{z}_{h}}{\bar{Z}_{h}}\right)+\omega_{2 h} \bar{y}_{h}^{*} \exp \left(\frac{\bar{z}_{h}-\bar{Z}_{h}}{\bar{Z}_{h}+\bar{z}_{h}}\right)\right.$ & Product-product-type exponential \\
\hline$\omega_{1 h}$ & $\omega_{2 h}$ & 1 & -1 & $T_{s t i}^{(7)}=\sum_{h=1}^{L} W_{h}\left[\omega_{1 h} \bar{y}_{h}^{*}\left(\frac{\bar{Z}_{h}}{\bar{z}_{h}}\right)+\omega_{2 h} \bar{y}_{h}^{*} \exp \left(\frac{\bar{z}_{h}-\bar{Z}_{h}}{\bar{Z}_{h}+\bar{z}_{h}}\right)\right.$ & Ratio-product-type exponential \\
\hline$\omega_{1 h}$ & $\omega_{2 h}$ & -1 & 1 & $T_{s t i}^{(8)}=\sum_{h=1}^{L} W_{h}\left[\omega_{1 h} \bar{y}_{h}^{*}\left(\frac{\bar{Z}_{h}}{\bar{Z}_{h}}\right)+\omega_{2 h} \bar{y}_{h}^{*} \exp \left(\frac{\bar{Z}_{h}-\bar{z}_{h}}{\bar{Z}_{h}+\bar{z}_{h}}\right)\right.$ & Product-ratio-type exponential \\
\hline
\end{tabular}


Table 3: Some members of $T_{\text {sti }}^{(1)}$ and $T_{\text {sti }}^{(3)}$

\begin{tabular}{|c|c|c|c|c|}
\hline & & & Ratio-type estimators & Ratio-type exponential \\
\hline$a_{h}$ & $b_{h}$ & $i$ & $T_{s t i}^{(1)}=\sum_{h=1}^{L} W_{h}\left[\omega_{1 h} \bar{y}_{h}^{*}\left(\frac{a_{h} \bar{X}_{h}+b_{h}}{a_{h} \bar{x}_{h}^{*}+b_{h}}\right)+\omega_{2 h} \bar{y}_{h}^{*}\right]$ & $T_{s t i}^{(3)}=\sum_{h=1}^{L} W_{h}\left[\omega_{1 h} \bar{y}_{h}^{*}+\omega_{2 h} \bar{y}_{h}^{*} \exp \left(\frac{a_{h}\left(\bar{X}_{h}-\dot{x}_{h}^{*}\right)}{a_{h}\left(\bar{X}_{h}+\dot{x}_{h}^{*}\right)+2 b_{h}}\right)\right.$ \\
\hline 1 & $C_{X h}$ & 1 & $T_{s t 1}^{(1)}=\sum_{h=1}^{L} W_{h}\left[\omega_{1 h} \bar{y}_{h}^{*}\left(\frac{\bar{X}_{h}+C_{X h}}{\overline{\hat{x}}_{h}^{*}+C_{X h}}\right)+\omega_{2 h} \bar{y}_{h}^{*}\right]$ & $T_{s t 1}^{(3)}=\sum_{h=1}^{L} W_{h}\left[\omega_{1 h} \bar{y}_{h}^{*}+\omega_{2 h} \bar{y}_{h}^{*} \exp \left(\frac{\bar{X}_{h}-\dot{\bar{x}}_{h}^{*}}{\bar{X}_{h}+\grave{x}_{h}^{*}+2 C_{X h}}\right)\right]$ \\
\hline$\beta_{2 h(X)}$ & $C_{X h}$ & 2 & $T_{s t 2}^{(1)}=\sum_{h=1}^{L} W_{h}\left[\omega_{1 h} \bar{y}_{h}^{*}\left(\frac{\beta_{2 h}(X) \bar{X}_{h}+C_{X h}}{\beta_{2 h}(X) \dot{\bar{x}}_{h}^{*}+C_{X h}}\right)+\omega_{2 h} \bar{y}_{h}^{*}\right]$ & $T_{s t 2}^{(3)}=\sum_{h=1}^{L} W_{h}\left[\omega_{1 h} \bar{y}_{h}^{*}+\omega_{2 h} \bar{y}_{h}^{*} \exp \left(\frac{\beta_{2 h(X)}\left(\bar{X}_{h}-\dot{\bar{x}}_{h}^{*}\right)}{\beta_{2 h(X)}\left(\bar{X}_{h}+\dot{\bar{x}}_{h}^{*}\right)+2 C_{X h}}\right)\right.$ \\
\hline 1 & $\rho_{Y X h}$ & 3 & $T_{s t 3}^{(1)}=\sum_{h=1}^{L} W_{h}\left[\omega_{1 h} \bar{y}_{h}^{*}\left(\frac{\bar{X}_{h}+\rho_{Y X h}}{\bar{x}_{h}^{*}+\rho_{Y X h}}\right)+\omega_{2 h} \bar{y}_{h}^{*}\right]$ & $T_{s t 3}^{(3)}=\sum_{h=1}^{L} W_{h}\left[\omega_{1 h} \bar{y}_{h}^{*}+\omega_{2 h} \bar{y}_{h}^{*} \exp \left(\frac{\bar{X}_{h}-\bar{x}_{h}^{*}}{\bar{X}_{h}+\dot{\bar{x}}_{h}^{*}+2 \rho_{Y X h}}\right)\right]$ \\
\hline$C_{X h}$ & $\rho_{Y X h}$ & 4 & $T_{s t 4}^{(1)}=\sum_{h=1}^{L} W_{h}\left[\omega_{1 h} \bar{y}_{h}^{*}\left(\frac{C_{X h} \bar{X}_{h}+\rho_{Y X h}}{C_{X h} \dot{\tilde{x}}_{h}^{*}+\rho_{Y X h}}\right)+\omega_{2 h} \bar{y}_{h}^{*}\right]$ & $T_{s t 4}^{(3)}=\sum_{h=1}^{L} W_{h}\left[\omega_{1 h} \bar{y}_{h}^{*}+\omega_{2 h} \bar{y}_{h}^{*} \exp \left(\frac{C_{X h}\left(\bar{X}_{h}-\dot{x}_{h}^{*}\right)}{C_{X h}\left(\bar{X}_{h}+\dot{x}_{h}^{*}\right)+2 \rho_{Y X h}}\right)\right]$ \\
\hline$S_{X h}$ & $\beta_{1 h(X)}$ & 5 & $T_{s t 5}^{(1)}=\sum_{h=1}^{L} W_{h}\left[\omega_{1 h} \bar{y}_{h}^{*}\left(\frac{S_{X h} \bar{X}_{h}+\beta_{1 h}(X)}{S_{X h} \dot{x}_{h}^{*}+\beta_{1 h}(X)}\right)+\omega_{2 h} \bar{y}_{h}^{*}\right]$ & $T_{s t 5}^{(3)}=\sum_{h=1}^{L} W_{h}\left[\omega_{1 h} \bar{y}_{h}^{*}+\omega_{2 h} \bar{y}_{h}^{*} \exp \left(\frac{S_{X h}\left(\bar{X}_{h}-\dot{\bar{x}}_{h}^{*}\right)}{S_{X h}\left(\bar{X}_{h}+\dot{x}_{h}^{*}\right)+2 \beta_{1 h(X)}}\right)\right]$ \\
\hline$H L_{h}$ & $C_{X h}$ & 6 & $T_{s t 6}^{(1)}=\sum_{h=1}^{L} W_{h}\left[\omega_{1 h} \bar{y}_{h}^{*}\left(\frac{H L_{h} \bar{X}_{h}+C_{X h}}{H L_{h} \dot{x}_{h}^{*}+C_{X h}}\right)+\omega_{2 h} \bar{y}_{h}^{*}\right]$ & $T_{s t 6}^{(3)}=\sum_{h=1}^{L} W_{h}\left[\omega_{1 h} \bar{y}_{h}^{*}+\omega_{2 h} \bar{y}_{h}^{*} \exp \left(\frac{H L_{h}\left(\bar{X}_{h}-\dot{x}_{h}^{*}\right)}{H L_{h}\left(\bar{X}_{h}+\dot{x}_{h}^{*}\right)+2 C_{X h}}\right)\right]$ \\
\hline$C_{X h}$ & $T M_{h}$ & 7 & $T_{s t 7}^{(1)}=\sum_{h=1}^{L} W_{h}\left[\omega_{1 h} \bar{y}_{h}^{*}\left(\frac{C_{X h} \bar{X}_{h}+T M_{h}}{C_{X h} \hat{x}_{h}^{*}+T M_{h}}\right)+\omega_{2 h} \bar{y}_{h}^{*}\right]$ & $T_{s t 7}^{(3)}=\sum_{h=1}^{L} W_{h}\left[\omega_{1 h} \bar{y}_{h}^{*}+\omega_{2 h} \bar{y}_{h}^{*} \exp \left(\frac{C_{X h}\left(\bar{X}_{h}-\dot{x}_{h}^{*}\right)}{C_{X h}\left(\bar{X}_{h}+\dot{x}_{h}^{*}\right)+2 T M_{h}}\right)\right]$ \\
\hline$M R_{h}$ & $\rho_{Y X h}$ & 8 & $T_{s t 8}^{(1)}=\sum_{h=1}^{L} W_{h}\left[\omega_{1 h} \bar{y}_{h}^{*}\left(\frac{M R_{h} \bar{X}_{h}+\rho_{Y X h}}{M R_{h} \dot{x}_{h}^{*}+\rho_{Y X h}}\right)+\omega_{2 h} \bar{y}_{h}^{*}\right]$ & $T_{s t 8}^{(3)}=\sum_{h=1}^{L} W_{h}\left[\omega_{1 h} \bar{y}_{h}^{*}+\omega_{2 h} \bar{y}_{h}^{*} \exp \left(\frac{M R_{h}\left(\bar{X}_{h}-\dot{\bar{x}}_{h}^{*}\right)}{M R_{h}\left(\bar{X}_{h}+\dot{\bar{x}}_{h}^{*}\right)+2 \rho_{Y X h}}\right)\right.$ \\
\hline$\rho_{Y X h}$ & $H L_{h}$ & 9 & $T_{s t 9}^{(1)}=\sum_{h=1}^{L} W_{h}\left[\omega_{1 h} \bar{y}_{h}^{*}\left(\frac{\rho_{Y X h} \bar{X}_{h}+H L_{h}}{\rho_{Y X h} \bar{x}_{h}^{*}+H L_{h}}\right)+\omega_{2 h} \bar{y}_{h}^{*}\right]$ & $T_{s t 9}^{(1)}=\sum_{h=1}^{L} W_{h}\left[\omega_{1 h} \bar{y}_{h}^{*}\left(\frac{\rho_{Y X h} \bar{X}_{h}+H L_{h}}{\rho_{Y X h} \hat{x}_{h}^{*}+H L_{h}}\right)+\omega_{2 h} \bar{y}_{h}^{*}\right]$ \\
\hline & & 10 & & um \\
\hline & & & 2 & 3 \\
\hline
\end{tabular}


Table 4: Data statistics population I

\begin{tabular}{|c|c|c|c|c|c|c|c|c|c|c|c|c|}
\hline \multicolumn{13}{|c|}{$h^{\text {th }}$ stratum } \\
\hline$h$ & \multicolumn{3}{|c|}{1} & \multicolumn{3}{|c|}{2} & \multicolumn{3}{|c|}{3} & \multicolumn{3}{|c|}{4} \\
\hline$X_{h}$ & \multicolumn{3}{|c|}{ rnorm $(1000,5,10)$, } & \multicolumn{3}{|c|}{ rnorm(1000, 4, 8), } & \multicolumn{3}{|c|}{$\operatorname{rnorm}(1000,4,9)$} & \multicolumn{3}{|c|}{$\operatorname{rnorm}(1000,3,7)$} \\
\hline$Y_{h}$ & \multicolumn{3}{|c|}{$X_{1}+\operatorname{rnorm}(1000,0,1)$} & \multicolumn{3}{|c|}{$X_{2}+\operatorname{rnorm}(1000,0,1)$} & \multicolumn{3}{|c|}{$X_{3}+\operatorname{rnorm}(1000,0,1)$} & \multicolumn{3}{|c|}{$X_{4}+\operatorname{rnorm}(1000,0,1)$} \\
\hline$y_{h}$ & \multicolumn{3}{|c|}{$Y_{1}+\operatorname{rnorm}(1000,1,3)$} & \multicolumn{3}{|c|}{$Y_{2}+\operatorname{rnorm}(1000,1,3)$} & \multicolumn{3}{|c|}{$Y_{3}+\operatorname{rnorm}(1000,1,3)$} & \multicolumn{3}{|c|}{$Y_{4}+\operatorname{rnorm}(1000,1,3)$} \\
\hline$x_{h}$ & \multicolumn{3}{|c|}{$X_{1}+\operatorname{rnorm}(1000,1,3)$} & \multicolumn{3}{|c|}{$X_{2}+\operatorname{rnorm}(1000,1,3)$} & \multicolumn{3}{|c|}{$X_{3}+\operatorname{rnorm}(1000,1,3)$} & \multicolumn{3}{|c|}{$X_{4}+\operatorname{rnorm}(1000,1,3)$} \\
\hline $\bar{Y}_{h}$ & \multicolumn{3}{|c|}{4.887} & \multicolumn{3}{|c|}{3.801} & \multicolumn{3}{|c|}{4.003} & \multicolumn{3}{|c|}{3.093} \\
\hline $\bar{X}_{h}$ & \multicolumn{3}{|c|}{4.911} & \multicolumn{3}{|c|}{3.757} & \multicolumn{3}{|c|}{4.044} & \multicolumn{3}{|c|}{3.128} \\
\hline$S_{Y h}^{2}$ & \multicolumn{3}{|c|}{97.315} & & 66.002 & & & 81.220 & & & 51.932 & \\
\hline$S_{X h}^{2}$ & & 97.036 & & & 64.658 & & & 80.508 & & & 50.357 & \\
\hline$S_{U h}^{2}$ & & 9.092 & & & 9.018 & & & 9.439 & & & 9.615 & \\
\hline$S_{V h}^{2}$ & & 9.590 & & & 9.233 & & & 9.735 & & & 9.124 & \\
\hline$\rho_{Y X h}$ & & 0.995 & & & 0.993 & & & 0.994 & & & 0.991 & \\
\hline$\beta_{1 h(X)}$ & & -0.06 & & & -0.11 & & & 0.09 & & & 0.08 & \\
\hline$\beta_{2 h(X)}$ & & -0.12 & & & -0.03 & & & 0.03 & & & -0.02 & \\
\hline$C_{X h}$ & & 2.006 & & & 2.140 & & & 2.219 & & & 2.269 & \\
\hline$H L_{h}$ & & 5.034 & & & 3.668 & & & 4.106 & & & 3.098 & \\
\hline$Q D_{h}$ & & 6.727 & & & 5.119 & & & 5.878 & & & 4.930 & \\
\hline$M R_{h}$ & & 4.262 & & & 4.292 & & & 2.885 & & & 3.045 & \\
\hline$T M_{h}$ & & 4.965 & & & 3.815 & & & 3.986 & & & 3.093 & \\
\hline $\begin{array}{l}\text { Non- } \\
\text { response }\end{array}$ & $10 \%$ & $15 \%$ & $20 \%$ & $10 \%$ & $15 \%$ & $20 \%$ & $10 \%$ & $15 \%$ & $20 \%$ & $10 \%$ & $15 \%$ & $20 \%$ \\
\hline$S_{Y(2) h}^{2}$ & 97.444 & 102.288 & 99.046 & 68.973 & 64.563 & 68.963 & 86.179 & 80.096 & 77.793 & 48.614 & 51.898 & 49.204 \\
\hline$S_{X(2) h}^{2}$ & 98.454 & 103.120 & 99.098 & 67.857 & 62.385 & 68.860 & 82.651 & 77.998 & 77.284 & 46.823 & 51.404 & 46.827 \\
\hline$S_{U(2) h}^{2}$ & 10.877 & 8.668 & 8.725 & 7.707 & 10.469 & 8.587 & 10.150 & 7.973 & 9.853 & 10.501 & 10.015 & 11.136 \\
\hline$S_{V(2) h}^{2}$ & 11.388 & 9.182 & 9.519 & 9.341 & 8.745 & 9.865 & 8.966 & 12.068 & 9.159 & 11.918 & 10.736 & 8.997 \\
\hline$\rho_{Y X(2) h}$ & 0.995 & 0.996 & 0.995 & 0.993 & 0.994 & 0.994 & 0.994 & 0.994 & 0.994 & 0.987 & 0.991 & 0.990 \\
\hline
\end{tabular}


Table 5: Data statistics population II

\begin{tabular}{|c|c|c|c|c|c|c|c|c|c|c|c|c|}
\hline$h$ & \multicolumn{3}{|c|}{1} & \multicolumn{3}{|c|}{2} & \multicolumn{3}{|c|}{3} & \multicolumn{3}{|c|}{4} \\
\hline$X_{h}$ & \multicolumn{3}{|c|}{$\operatorname{rnorm}(1000,5,10)$} & \multicolumn{3}{|c|}{ rnorm $(1200,4,8)$} & \multicolumn{3}{|c|}{$\operatorname{rnorm}(1300,4,9)$} & \multicolumn{3}{|c|}{ rnorm(1500, 3, 7), } \\
\hline$Y_{h}$ & \multicolumn{3}{|c|}{$X_{1}+\operatorname{rnorm}(1000,0,1)$} & \multicolumn{3}{|c|}{$X_{2}+\operatorname{rnorm}(1200,0,1)$} & \multicolumn{3}{|c|}{$X_{3}+\operatorname{rnorm}(1300,0,1)$} & \multicolumn{3}{|c|}{$X_{4}+\operatorname{rnorm}(1500,0,1)$} \\
\hline$y_{h}$ & \multicolumn{3}{|c|}{$Y_{1}+\operatorname{rnorm}(1000,1,3)$} & \multicolumn{3}{|c|}{$Y_{2}+\operatorname{rnorm}(1200,1,3)$} & \multicolumn{3}{|c|}{$Y_{3}+\operatorname{rnorm}(1300,1,3)$} & \multicolumn{3}{|c|}{$Y_{4}+\operatorname{rnorm}(1500,1,3)$} \\
\hline$x_{h}$ & \multicolumn{3}{|c|}{$X_{1}+\operatorname{rnorm}(1000,1,3)$} & \multicolumn{3}{|c|}{$X_{2}+\operatorname{rnorm}(1200,1,3)$} & \multicolumn{3}{|c|}{$X_{3}+\operatorname{rnorm}(1300,1,3)$} & \multicolumn{3}{|c|}{$X_{4}+\operatorname{rnorm}(1500,1,3)$} \\
\hline$n_{h}$ & \multicolumn{3}{|c|}{200} & \multicolumn{3}{|c|}{240} & \multicolumn{3}{|c|}{260} & \multicolumn{3}{|c|}{300} \\
\hline $\bar{Y}_{h}$ & \multicolumn{3}{|c|}{5.157} & \multicolumn{3}{|c|}{3.649} & \multicolumn{3}{|c|}{3.563} & \multicolumn{3}{|c|}{2.765} \\
\hline $\bar{X}_{h}$ & \multicolumn{3}{|c|}{5.148} & \multicolumn{3}{|c|}{3.686} & & 3.554 & & & 2.756 & \\
\hline$S_{\underline{Y} h}^{2}$ & & 99.459 & & & 60.788 & & & 88.185 & & & 49.353 & \\
\hline$S_{X h}^{2}$ & & 98.151 & & & 59.113 & & & 86.809 & & & 48.277 & \\
\hline$S_{U h}^{2}$ & & 9.152 & & & 8.882 & & & 8.829 & & & 9.426 & \\
\hline$S_{V h}^{2}$ & & 9.666 & & & 8.821 & & & 9.003 & & & 8.775 & \\
\hline$\rho_{Y X h}$ & & 0.995 & & & 0.992 & & & 0.994 & & & 0.990 & \\
\hline$\beta_{1 h(X)}$ & & -0.05 & & & 0.10 & & & -0.05 & & & 0.03 & \\
\hline$\beta_{2 h(X)}$ & & 0.01 & & & 0.05 & & & 0.07 & & & 0.07 & \\
\hline$C_{X h}$ & & 1.924 & & & 2.086 & & & 2.622 & & & 2.521 & \\
\hline$H L_{h}$ & & 5.125 & & & 3.671 & & & 3.468 & & & 2.605 & \\
\hline$Q D_{h}$ & & 6.765 & & & 5.093 & & & 6.172 & & & 4.714 & \\
\hline$M R_{h}$ & & 4.781 & & & 4.493 & & & 2.572 & & & 2.685 & \\
\hline$T M_{h}$ & & 5.196 & & & 3.630 & & & 3.569 & & & 2.734 & \\
\hline $\begin{array}{l}\text { Non- } \\
\text { response }\end{array}$ & $10 \%$ & $15 \%$ & $20 \%$ & $10 \%$ & $15 \%$ & $20 \%$ & $10 \%$ & $15 \%$ & $20 \%$ & $10 \%$ & $15 \%$ & $20 \%$ \\
\hline$S_{Y(2) h}^{2}$ & 113.600 & 112.505 & 107.682 & 59.723 & 65.300 & 67.144 & 82.390 & 76.975 & 78.004 & 51.278 & 50.815 & 51.802 \\
\hline$S_{X(2) h}^{2}$ & 112.810 & 111.589 & 105.596 & 60.529 & 65.222 & 64.166 & 81.073 & 78.085 & 78.551 & 50.360 & 49.828 & 50.710 \\
\hline$S_{U(2) h}^{2}$ & 9.279 & 8.273 & 8.405 & 8.980 & 9.110 & 9.794 & 8.216 & 8.509 & 7.170 & 9.561 & 9.506 & 11.108 \\
\hline$S_{V(2) h}^{2}$ & 10.708 & 10.735 & 10.324 & 10.005 & 10.349 & 10.193 & 9.362 & 8.300 & 8.704 & 9.307 & 8.843 & 9.860 \\
\hline$\rho_{Y X(2) h}$ & 0.996 & 0.995 & 0.994 & 0.993 & 0.992 & 0.992 & 0.994 & 0.993 & 0.994 & 0.991 & 0.990 & 0.991 \\
\hline
\end{tabular}


Table 6: Percent relative efficiencies of the competing estimators w.r.t. $\bar{y}_{S t(H H)}^{*}$ for population I (10 \% non-response)

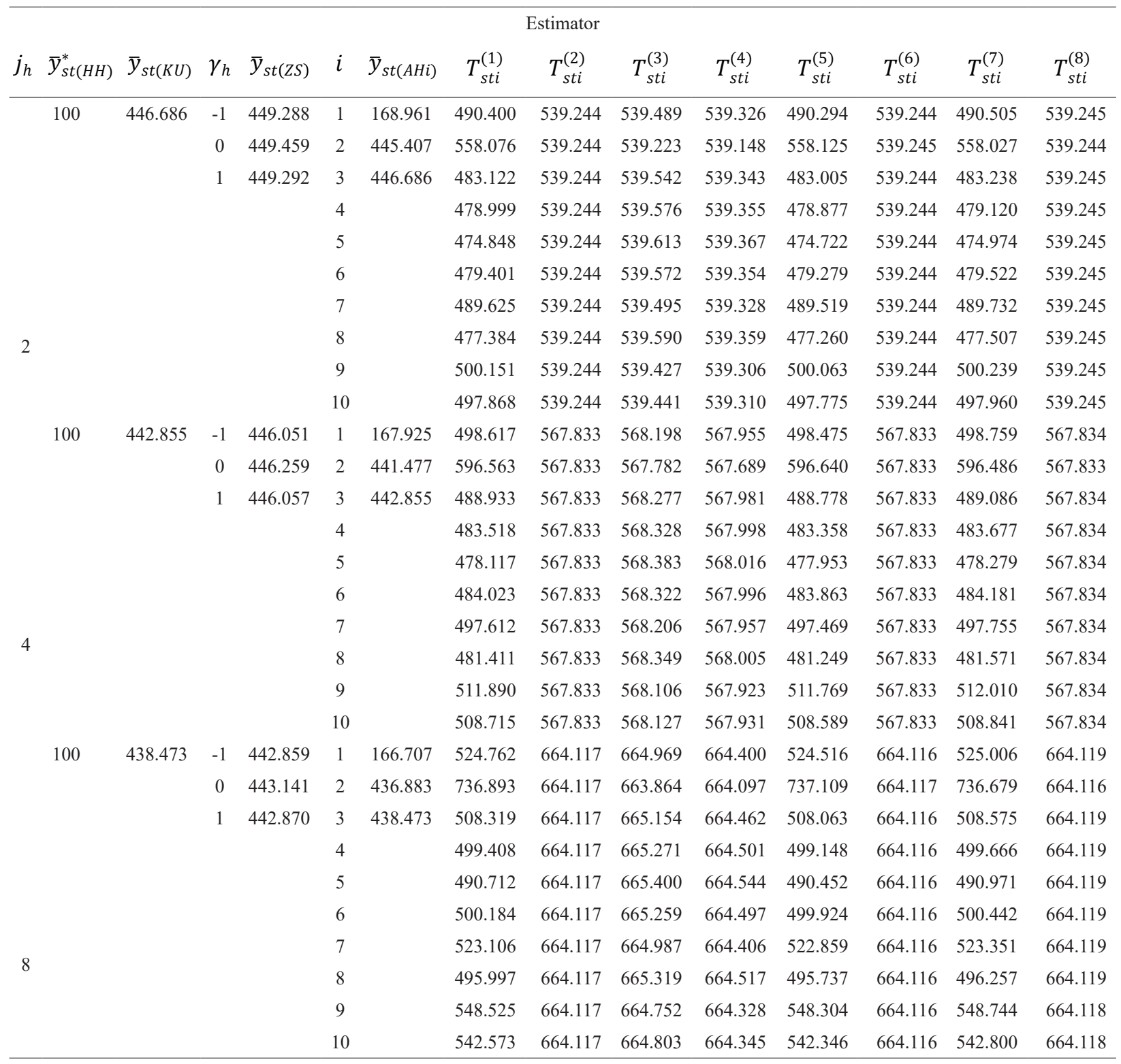


Table 7: Percent relative efficiencies of the competing estimators w.r.t. $\bar{y}_{s t(H H)}^{*}$ for population II (10\% non-response)

\begin{tabular}{|c|c|c|c|c|c|c|c|c|c|c|c|c|c|c|}
\hline \multicolumn{15}{|c|}{ Estimator } \\
\hline$j_{h}$ & $\bar{y}_{s t(H H)}^{*}$ & $\bar{y}_{s t(K U)}$ & $\gamma_{h}$ & $\bar{y}_{s t(Z S)}$ & $i$ & $\bar{y}_{s t(A H i)}$ & $T_{s t i}^{(1)}$ & $T_{s t i}^{(2)}$ & $T_{\text {sti }}^{(3)}$ & $T_{s t i}^{(4)}$ & $T_{s t i}^{(5)}$ & $T_{s t i}^{(6)}$ & $T_{s t i}^{(7)}$ & $T_{s t i}^{(8)}$ \\
\hline \multirow{11}{*}{2} & 100 & 449.713 & -1 & 452.051 & 1 & 169.061 & 492.206 & 533.996 & 534.175 & 534.056 & 492.125 & 533.996 & 492.286 & 533.997 \\
\hline & & & 0 & 452.207 & 2 & 448.073 & 528.951 & 533.996 & 534.128 & 534.067 & 528.941 & 533.996 & 528.962 & 533.996 \\
\hline & & & 1 & 452.054 & 3 & 449.713 & 484.637 & 533.996 & 534.217 & 534.070 & 484.547 & 533.996 & 484.728 & 533.997 \\
\hline & & & & & 4 & & 480.487 & 533.996 & 534.243 & 534.079 & 480.392 & 533.996 & 480.582 & 533.997 \\
\hline & & & & & 5 & & 476.737 & 533.996 & 534.271 & 534.088 & 476.637 & 533.996 & 476.836 & 533.997 \\
\hline & & & & & 6 & & 481.964 & 533.996 & 534.235 & 534.075 & 481.869 & 533.996 & 482.058 & 533.997 \\
\hline & & & & & 7 & & 489.614 & 533.996 & 534.184 & 534.058 & 489.531 & 533.996 & 489.697 & 533.997 \\
\hline & & & & & 8 & & 479.412 & 533.996 & 534.252 & 534.082 & 479.315 & 533.996 & 479.508 & 533.997 \\
\hline & & & & & 9 & & 499.248 & 533.996 & 534.133 & 534.043 & 499.180 & 533.996 & 499.316 & 533.997 \\
\hline & & & & & 10 & & 497.682 & 533.996 & 534.141 & 534.044 & 497.612 & 533.996 & 497.753 & 533.997 \\
\hline & 100 & 449.635 & -1 & 452.499 & 1 & 168.209 & 505.438 & 566.401 & 566.676 & 566.492 & 505.326 & 566.401 & 505.550 & 566.402 \\
\hline \multirow{9}{*}{4} & & & 0 & 452.692 & 2 & 448.030 & 558.948 & 566.401 & 566.468 & 566.400 & 558.933 & 566.401 & 558.963 & 566.401 \\
\hline & & & 1 & 452.504 & 3 & 449.635 & 495.186 & 566.401 & 566.740 & 566.514 & 495.061 & 566.401 & 495.310 & 566.402 \\
\hline & & & & & 4 & & 489.626 & 566.401 & 566.780 & 566.527 & 489.497 & 566.401 & 489.755 & 566.402 \\
\hline & & & & & 5 & & 484.602 & 566.401 & 566.822 & 566.541 & 484.469 & 566.401 & 484.735 & 566.402 \\
\hline & & & & & 6 & & 491.504 & 566.401 & 566.769 & 566.524 & 491.375 & 566.401 & 491.632 & 566.402 \\
\hline & & & & & 7 & & 502.174 & 566.401 & 566.688 & 566.497 & 502.059 & 566.401 & 502.288 & 566.402 \\
\hline & & & & & 8 & & 488.131 & 566.401 & 566.793 & 566.532 & 488.000 & 566.401 & 488.262 & 566.402 \\
\hline & & & & & 9 & & 515.510 & 566.401 & 566.613 & 566.472 & 515.414 & 566.401 & 515.606 & 566.402 \\
\hline & & & & & 10 & & 513.338 & 566.401 & 566.623 & 566.474 & 513.238 & 566.401 & 513.437 & 566.402 \\
\hline \multirow{10}{*}{8} & 100 & 449.570 & -1 & 453.488 & 1 & 167.203 & 538.909 & 665.877 & 666.543 & 666.098 & 538.704 & 665.877 & 539.113 & 665.879 \\
\hline & & & 0 & 453.753 & 2 & 447.979 & 649.250 & 665.877 & 665.970 & 665.847 & 649.217 & 665.877 & 649.283 & 665.878 \\
\hline & & & 1 & 453.497 & 3 & 449.570 & 521.153 & 665.877 & 666.694 & 666.149 & 520.936 & 665.877 & 521.369 & 665.879 \\
\hline & & & & & 4 & & 511.803 & 665.877 & 666.789 & 666.181 & 511.582 & 665.877 & 512.023 & 665.879 \\
\hline & & & & & 5 & & 503.421 & 665.877 & 666.888 & 666.214 & 503.199 & 665.877 & 503.643 & 665.879 \\
\hline & & & & & 6 & & 514.693 & 665.877 & 666.766 & 666.173 & 514.472 & 665.877 & 514.913 & 665.879 \\
\hline & & & & & 7 & & 533.913 & 665.877 & 666.566 & 666.107 & 533.707 & 665.877 & 534.119 & 665.879 \\
\hline & & & & & 8 & & 509.171 & 665.877 & 666.823 & 666.192 & 508.949 & 665.877 & 509.392 & 665.879 \\
\hline & & & & & 9 & & 558.191 & 665.877 & 666.383 & 666.047 & 558.007 & 665.877 & 558.373 & 665.878 \\
\hline & & & & & 10 & & 554.145 & 665.877 & 666.411 & 666.055 & 553.958 & 665.877 & 554.332 & 665.879 \\
\hline
\end{tabular}

